\title{
SPECTROSCOPICAL ANALYSIS OF $\delta$ SCUTI STARS
}

\author{
E. SOLANO \\ VILSPA-INSA. P.O. Box 50727. 28080 Madrid (Spain) \\ and \\ J. FERNLEY \\ VILSPA-ESA. P.O. Box 50727. 28080 Madrid (Spain)
}

$\delta$ Scuti are pulsating stars with a characteristic period of several hours, lying in the A2-F5 spectral range and placed on or a little above the main sequence. Unlike the well-known "classical" RR Lyrae or Cepheids, which show a homogeneous pulsational behaviour (i.e. radial modes with large amplitudes), $\delta$ Scuti stars have peculiarities: their amplitudes vary from millimagnitudes to tenths of magnitude and their pulsation modes can be both radial and non-radial.

$67 \delta$ Scuti stars have been observed in different campaigns. The aim of this work is to calculate the physical parameters associated with each $\operatorname{star}\left(T_{\text {eff }}, v \cdot \sin i,[\mathrm{M} / \mathrm{H}]\right)$ using different methods to get values as accurate as possible and to establish possible correlations between these parameters and the pulsational behaviour of the stars.

\section{Measurement of rotation}

Two methods of measuring rotational velocities have been studied: The first, described in Sletteback (1975), is based on the existing linear relationship between the full width at half maximum of a spectral line and the rotational velocity of the star while the second (Gray,1975,1992) uses properties of the Fourier transform of the rotation profile to calculate rotational velocities. After different tests, we decided to used the second one because it provides more accurate values and it allows us to distinguish between rotation and macroturbulence.

The influence of rotation on pulsation has been discussed by some authors (e.g. Mc Namara,1985). The idea pointed out by this author that large pulsational amplitudes are only present in stars with rotational velocities $\leq 40 \mathrm{kms}^{-1}$ is confirmed with our larger sample of spectra. A theoretical explanation can be found in Dziembowski (1988).

\section{Measurement of effective temperature}

The $H_{\beta}$ line profile is well known to be a good indicator of the effective temperature in the range where the $\delta$ Scuti lie because it is not affected 
by the gravity . A grid of $H_{\beta}$ synthetic profiles were built using Kurucz's models (1979).

The values calculated in this way have been compared with those ones from photometric calibrations. As each one of them is based on different color indexes $\left(c_{1}-(b-y), c_{1}-\beta\right)$ and on different standards stars, comparisons with our set of values will give information about which calibrations show systematic errors (i.e. Philip \& Relyea, 1979) and which agree assuming a random error of $\pm 100 \mathrm{~K}$ (i.e. Moon \& Dworetsky, 1985).

\section{Spectral restoration and metallicity calculation}

Due to instrumental limitations or to phenomena which take place in the proper star (rotation, turbulences...), the observed spectrum can appear broadened with the subsequent loss in contrast. Essential in the metallicity calculation is to get values of equivalent width as accurate as possible; therefore some spectral restoration techniques have to be used. After different tests, it was seen that the most suitable technique for our purposes is the one developed by Jansson (1970) based on the non-linear constrained algorithms.

The metallicity calculations and comparisons with values from photometric calibrations are still in progress.

\section{References}

Dziembowsky,W: 1988, Acta Astronomica 38, 61.

Gray,D.F.: 1975, Astrophysical Journal 202, 148.

Gray,D.F.: 1992, The observation and analysis of stellar photospheres, University Press:

Cambridge, 380.

Jansson P.: 1984, Deconvolution. Applications in spectroscopy, Academic Press: London, 183.

Mc Namara D.H.: 1985, P.A.S.P. 97, 715.

Moon T., Dworetsky M.: 1985, M.N.R.A.S. 217, 305.

Philip A., Relyea L.: 1979, Astronomical Journal 84, 1743.

Sletteback A.: 1985, A strophysical Journal Supplement 29., 137. 